\title{
Atelier de réécriture et critique littéraire en acte à l'université
}

Violaine Houdart-Mérot

\section{OpenEdition}

1 Journals

\section{Édition électronique}

URL : http://journals.openedition.org/recherchestravaux/332

DOI : 10.4000/recherchestravaux.332

ISSN : 1969-6434

Éditeur

UGA Éditions/Université Grenoble Alpes

\section{Édition imprimée}

Date de publication : 15 décembre 2008

Pagination : 125-137

ISBN : 978-2-84310-131-1

ISSN : 0151-1874

\section{Référence électronique}

Violaine Houdart-Mérot, « Atelier de réécriture et critique littéraire en acte à l'université », Recherches \& Travaux [En ligne], 73 | 2008, mis en ligne le 15 juin 2010, consulté le 08 septembre 2020. URL : http:// journals.openedition.org/recherchestravaux/332; DOI : https://doi.org/10.4000/recherchestravaux 332 
Violaine HOUdART-MÉrot

Université de Cergy-Pontoise

Pôle «Transmission littéraire et dialogues intertextuels et interculturels»

\section{Atelier de réécriture et critique littéraire en acte à l'université}

Dans un contexte de crise des études de lettres, alors que l'on s'inquiète de la désaffection et de l'échec des étudiants de Lettres, il apparaît de plus en plus urgent de repenser la place qu'y occupe l'écriture. Le peu d'importance accordée aux travaux écrits (même métatextuels) et l'absence d'une écriture à dimension littéraire dans une discipline qui se veut artistique, expliquent en partie les abandons en cours de licence et la difficulté pour bien des étudiants qui continuent en master à mener à terme leurs travaux de recherche. Renforcer le rôle de l'écriture, et plus généralement de l'expression, écrite ou orale, ne plus la cantonner à la seule écriture critique pourrait être un des moyens de redonner du sens à ces études et, indirectement, de permettre aux étudiants une meilleure maitrise également dans le domaine de l'écriture critique.

Nous partirons de l'analyse d'une pratique d'écriture mise en œuvre depuis maintenant six ans à l'université de Cergy-Pontoise auprès d'étudiants de troisième année de licence de Lettres ${ }^{1}$.

Il s'agit d'un atelier d'écriture, actuellement optionnel, centré exclusivement sur des démarches intertextuelles, et introduisant une importante dimension réflexive et théorique.

L'atelier comprend douze séances de trois heures durant lesquelles des moments d'écriture individuelle alternent avec des moments de lecture à voix

I. Cette expérience fait elle-même suite à la publication d'un ouvrage à la fois théorique et pratique, à destination des lycéens mais aussi des étudiants de lettres, Réécriture et écriture d'invention au lycée, Hachette «Profession enseignant», 2004. 
haute (donnant lieu à des analyses et des propositions de remaniements) et avec des pauses théoriques qui se situent tantôt en début tantôt en fin de séances. Pour chaque proposition d'écriture est demandée une deuxième version (reprise de la première version ou nouvelle version), produite le plus souvent en dehors des séances de cours, à partir des conseils donnés soit par l'enseignante soit par les autres étudiants qui jouent alors le rôle de comité de lecture.

Les séances sont conçues selon une double exigence, celle d'aborder les principaux genres littéraires (conte, nouvelle, poésie, monologue et dialogue de théâtre, écritures brèves comme la maxime...), mais aussi les principales relations intertextuelles ${ }^{2}$, des plus massives aux plus occultes, depuis l'insertion de citation ou le centon jusqu'aux relations architextuelles, en passant par les multiples procédés hypertextuels, tels que traduction, adaptation, transposition, parodie, travestissement burlesque, pastiches d'auteurs, de mouvements littéraires et même pastiches de genres ${ }^{3}$. L'intertextualité y est donc abordée à la fois comme outil de lecture et comme outil d'écriture.

Cette option intitulée «écriture d'invention et réécriture» donne lieu à une double évaluation : tout d'abord celle d'un dossier contenant l'ensemble des écrits produits durant les séances, ainsi que les nouvelles versions rédigées en dehors de l'atelier et enfin des commentaires critiques personnels sur ces productions, pouvant intégrer des réflexions sur les travaux des autres étudiants ou sur les démarches proposées. Outre ce compte-rendu (équivalent d'un devoir à la maison), un devoir sur table a lieu en fin de semestre. Il s'agit d'une sorte d'essai, bilan personnel, réflexion sur la littérature et sur la place de l'intertextualité dans les processus d'écriture, étayée sur l'expérience de l'atelier, les textes littéraires rencontrés au cours des séances et les divers apports théoriques.

Ces séances d'écriture poursuivent donc plusieurs objectifs, mais atteignent par inadvertance d'autres objectifs, qui n'étaient pas prévus a priori et qui se sont avérés essentiels. Ces effets inattendus, ce sont les propres analyses des étudiants qui en ont révélé l’importance.

2. Au sens où J. Kristeva introduit la notion dans Semeiotikè, sens repris ensuite par L. Jenny, M. Riffaterre, ou P.-M. de Biasi.

3. Pour la notion de pastiche de genre, voir mon article «Le pastiche de genre, entre feinte et réflexivité», Poétiques de la parodie et du pastiche de I850 à nos jours (C. Dousteyssier-Khoze et Fl. Place-Verghnes éd.), Éditions Peter Lang, 2006, p. 289-299. 


\section{Diversifier l'écriture, faciliter l'accès à l'écriture}

Le premier objectif est d'abord, comme pour un grand nombre d'ateliers d'écriture ${ }^{4}$ d'explorer l'écriture littéraire et de faciliter l'accès à l'écriture par les dispositifs mis en place : propositions élaborées selon un déroulement progressif, davantage encadré dans les premières séances; contraintes qui libèrent l'écriture; stimulation due à l'écriture en groupe et au temps limité; encadrement et aide de l'enseignant; lecture à haute voix du texte écrit (avec une possibilité de passer son tour éventuellement, la lecture devant témoins étant une contrainte intimidante dans un premier temps, mais très bénéfique à terme).

Nous laisserons de côté l'intérêt, pour toute personne, d'avoir accès à une écriture personnelle et créative, indépendamment de tout projet professionnel.

Nous passerons rapidement aussi sur l'importance, pour des étudiants de lettres, de se frotter à l'écriture littéraire, dans toute sa diversité, d'acquérir ainsi une plus grande maîtrise de l'expression écrite, une plus grande aisance, un plus grand plaisir, sachant que beaucoup d'entre eux, dans leur vie professionnelle, seront confrontés à des modes d'écriture beaucoup plus diversifiés que la seule écriture de commentaire, qu'ils s'orientent ensuite vers une carrière journalistique ou bien vers les métiers du livre, de la communication, du tourisme ou de l'édition, sans parler des métiers de l'enseignement, s'il est vrai qu'il importe d'avoir soi-même une expérience d'écriture pour pouvoir à son tour faire écrire, et faire écrire toutes sortes de textes.

Mais cet entraînement à l'écriture littéraire, tel qu'il est conçu ici, a pour ambition conjointe de faire non seulement, selon la formule de Marcel Proust à propos des pastiches d'écrivains', «de la critique littéraire en acte» mais aussi de la théorie littéraire et de l'histoire littéraire en acte, par le truchement de l'écriture elle-même. Autrement dit, l'atelier d'écriture adossé aux textes littéraires, conçu comme atelier de réécriture, et intégrant une part de réflexivité, permet, à terme, de retrouver l'écriture critique, par certains détours, mais avec une efficacité parfois bien plus forte. À condition néanmoins de veiller à certains principes qui peuvent se résumer à trois mots : intertextualité, réflexivité et remaniement des premières versions.

4. Voir par exemple l'ouvrage déjà ancien mais toujours précieux d'A. André, Babel heureuse. L'Atelier d'écriture au service de la création littéraire, Syros, 1989.

5. Mais, on l'aura compris, cet atelier ne se cantonne pas aux seuls pastiches d'écrivains et les autres formes d'hypertextualité permettent également de faire de la critique littéraire en acte. 


\section{Découvrir les paradoxes de l'écriture littéraire}

Aborder l'écriture par l'intertextualité est une manière de rendre visible un processus d'écriture fondamental, mais qui n'est pas toujours conscient, que «tout texte se construit comme une mosaïque de citations ${ }^{6}{ }$ et que l'on écrit toujours à partir des écrits des autres, quand bien même il ne s'agit pas de processus de réécritures affichés comme tels. Ainsi, les relations d'ordre taxinomique qu'une œuvre entretient avec des genres ou des sous-genres relèvent également de l'intertextualité telle que la définissent Julia Kristeva ou Laurent Jenny ${ }^{7}$ et la mise en évidence de ces liens habituellement occultes joue un rôle aussi bien pour l'écriture que pour la lecture.

De la sorte, selon un paradoxe inhérent à la littérature, on s'aperçoit que l'originalité n'est pas dissociable de l'imitation, conçue comme appropriation et transformation ou transgression de textes, de discours ou de genres existants (et non comme simple application de règles, ce à quoi l'on a souvent tendance à réduire l'imitation). Au contraire, l'imitation consciente et délibérée peut devenir une forme d'exorcisme ${ }^{8}$ contre l'imitation inconsciente et non maittrisée. Plus précisément, il s'agit, dans cet atelier d'«écriture intertextuelle» de proposer aux étudiants des travaux d'écriture qui s'inspirent de démarches intertextuelles déjà mises en œuvre par des écrivains : par exemple insérer une citation venue d'ailleurs dans son texte en la détournant de son sens (comme Molière peut le faire en reprenant tel ou tel vers de Corneille); ou bien faire parler un personnage, au théâtre ou dans un roman, en pastichant tel ou tel discours social ou littéraire : parler paysan (Maupassant...), discours patriotique (Céline) ou stéréotypes romantiques (Flaubert...); s'inspirer du dilemme cornélien pour écrire une parodie de monologue de théâtre (Tardieu), faire un travestissement burlesque de la descente aux enfers d'Énée dans l'Odyssée

6. J. Kristeva, Sémeiotikè, Seuil, I969, p.145-146.

7. Voir L. Jenny : «Hors de l'intertextualité, l’œuvre littéraire serait tout simplement imperceptible, au même titre que la parole d'une langue encore inconnue. De fait, on ne saisit le sens et la structure d'une œuvre littéraire que dans son rapport à des archétypes, eux-mêmes abstraits de longues séries de textes dont ils sont en quelque sorte l'invariant.», «La stratégie de la forme», Poétique, $\mathrm{n}^{\circ} 27, \mathrm{1} 976$, p. 257.

8. Voir M. Proust : «Je ne saurais trop recommander aux écrivains la vertu purgative, exorcisante du pastiche. Quand on vient de finir un livre, non seulement on voudrait continuer à vivre avec ses personnages, avec Mme de Bauséant, avec Frédéric Moreau, mais encore notre voix intérieure, qui a été disciplinée pendant toute la durée de la lecture à suivre le rythme d'un Balzac, d'un Flaubert voudrait continuer à parler comme eux. Il faut la laisser faire un moment, laisser la pédale prolonger le son, c'est-à-dire faire un pastiche volontaire, pour pouvoir après cela, redevenir original, ne pas faire toute sa vie du pastiche involontaire», Contre Sainte Beuve, Essais et articles, Après la guerre, "À propos du «style» de Flaubert», Gallimard, Bibliothèque de la Pléiade, I97ı, p. 594. 
d'Homère, tout comme Rabelais dans son Pantagruel. Ou enfin, partir du «Dormeur éveillé» des Mille et une nuits, de sa réécriture oblique par Proust au début de la Recherche 9 , pour écrire sur l'état de dormeur éveillé propre au moment du réveil. Ce faisant, on prend conscience que l'interprétation d'un texte littéraire s'appuie presque toujours sur une lecture intertextuelle : comment interpréter la descente aux enfers d'Épistémon chez Rabelais si l'on n'y décèle pas sa dimension parodique et carnavalesque et la pluralité de lectures qui en découle? Ainsi, faire écrire un étudiant en lui proposant d'exploiter un processus intertextuel est une manière de lui faire comprendre de l'intérieur, à travers une expérience d'écriture, le lien entre intertextualité et interprétation, entre intertextualité et littérarité d'une œuvre.

L'autre expérience que font les étudiants en pratiquant ainsi «l'imitation» et en écrivant à partir des écrits des autres est celle de la diversité étonnante des productions et de leur dimension fortement personnelle et impliquée, alors même que les propositions ne les amènent pas a priori à parler d'euxmêmes, qu'il s'agisse de faire un portrait à la manière de Céline ou au contraire de La Bruyère, de réécrire le mythe de Pygmalion ou le mythe de Babel en procédant à des changements spatio-temporels ou axiologiques, ou encore d'écrire un monologue de théâtre à partir d'un poème de Tardieu. Autrement dit, la contrainte, par un apparent paradoxe, libère et rend possible l'expression de soi. Les étudiants prennent ainsi conscience, de l'intérieur, par cette expérience d'écriture collective, du fait que l'expression personnelle s'accommode fort bien, et même parfois a besoin de détours.

Enfin, chacun peut constater durant ces séances d'écriture collective combien l'écriture peut vous engager dans des directions totalement inattendues. Il en va pour l'écriture créative comme pour toute forme d'élaboration intellectuelle. L'analyse de Clément Rosset est bien sûr applicable à toute forme d'écriture : «Il n'y a pensée qu'à partir du moment où celle-ci se formule, c'est-à-dire se constitue par la réalité des mots ${ }^{10}$.»

Ainsi, en écrivant un poème à partir de la reproduction d'une œuvre picturale, telle étudiante s'est surprise elle-même à aborder la question, longtemps douloureuse pour elle, de la couleur de sa peau et de la difficulté précisément d'en parler. Telle autre vit surgir la figure d'un grand-père mort par le biais d'une lettre à un inconnu. Le détour de la contrainte rend possible l'aveu de secrets, précisément parce que cet aveu demeure indirect et pudique. Il

9. Voir à ce sujet mon étude sur «Proust Dormeur éveillé ou comment surseoir à l'arrêt de mort», Les Mille et Une nuits dans l'imaginaire du XX siècle, Chr. Chaulet-Achour coord., L'Harmattan «Études transnationales francophones et comparées», 2005.

ı. Cl. Rosset, Le Choix des mots, Minuit, 1995 . 
s'agit bien d'une découverte essentielle, celle de la primauté et du pouvoir des mots: «les mots qui vont surgir savent de nous ce que nous ignorons d'eux ${ }^{11} . »$ Le travail sur le centon est particulièrement révélateur à ce sujet. Lorsque, à partir d'un même corpus de poèmes en alexandrins, les étudiants élaborent leur propre poème, fait d'hémistiches cousus ensemble, ils constatent eux-mêmes qu'ils produisent des poèmes d'une étonnante diversité et dont on pourrait retrouver chaque auteur tant ces centons leur ressemblent.

\section{Faire de l'histoire littéraire en acte}

Dans la mesure où il explore la diversité des processus intertextuels, cet atelier d'écriture permet aussi de faire de l'histoire littéraire en acte, à partir d'une nouvelle approche de l'histoire littéraire. En effet, le fait d'appréhender le texte littéraire comme une productivité ${ }^{12}$ où interviennent moins les rapports entre le texte et le réel (et par conséquent l'Histoire) qu'entre ce texte et les autres textes qu'il absorbe, qu'il transforme ou avec lesquels il entre en dialogue, conduit à réintroduire l'histoire littéraire, mise à mal par la critique formaliste et la perspective du texte clos sur lui-même. L'histoire littéraire ainsi conçue subit donc un déplacement du contexte historique à l'histoire des formes et des idées. Ainsi que l'explique Sophie Rabau, l'intertextualité suppose en effet «un déplacement dans la conception même de l'interprétation littéraire» : là où l'on interprétait le texte en fonction de causes extérieures, selon un axe logico-temporel, «il va falloir l'interpréter en fonction d'un réseau où il se trouve pris $^{13}$.»

Dans cette optique, on peut définir l'histoire littéraire comme histoire des opérations par lesquelles les textes réécrivent d'autres textes et les genres engendrent d'autres genres, dans un mouvement qui est à la fois d'interprétation, d'imitation transformatrice et de transgression incessantes. Les textes littéraires sont alors appréhendés non seulement comme signes et discours sur l'histoire (et non simples produits de l'histoire comme c'était le cas dans une conception traditionnelle de l'histoire littéraire), mais aussi comme réécritures (ou relectures) des textes qui les ont précédés ou qui les entourent. Une telle approche de l'histoire littéraire qui n'évacue pas les textes mais s'appuie

I I. R. Char, «Ma feuille vineuse», I983, p. 534.

I 2. Voir R. Barthes: "Tout texte est un intertexte. C'est tout le langage, antérieur et contemporain, qui vient au texte, non selon la voie d'une filiation repérable, d'une imitation volontaire, mais selon celle d'une dissémination - image qui assure au texte le statut, non d'une reproduction, mais d'une productivité.», «Texte [Théorie du]», Encyclopaedia universalis, I975.

I3. S. Rabau, L'Intertextualité, GF Flammarion, 2002, p. 33. 
sur eux, donne accès à l'historicité des œuvres, sans pour autant escamoter la dimension interprétative. Elle permet également de réintroduire le lecteur, puisque le texte est conçu comme productivité dans laquelle le récepteur a sa part à jouer dans l'élaboration du sens et l'accès à la signification.

Cette histoire littéraire en acte, mise au cœur de l'atelier d'écriture, peut se décliner dans plusieurs directions et notamment celle de l'histoire des idées, des mouvements littéraires et des genres. Nous ne nous attarderons pas sur l'histoire des idées, relativement facile à déceler à travers les réécritures de mythes ou de légendes : la confrontation des diverses versions d'un même récit permet de voir en quoi chaque reprise est une réinterprétation révélatrice des valeurs et des idéologies d'une époque : ainsi le héros éponyme de l'CEdipe de Corneille, étonnamment chrétien et défenseur du libre-arbitre est bien différent de l'CEdipe Roi de Sophocle ou de l'CEdipe de Sénèque. De même la réécriture par La Fontaine du récit des amours de Psyché et de Cupidon est révélatrice d'une vision épicurienne (voire libertine) qui n'existait pas dans la version d'Apulée. Si l'on compare le dénouement du conte chez La Fontaine et son hypotexte apuléen, on voit bien que l'hymne à Volupté, petite fille née des amours de Cupidon et de Psyché, devenue immortelle après avoir été poursuivie de la jalousie de Vénus, entrelace plusieurs hypotextes et que le plus signifiant, ici, est l'hymne à Vénus de Lucrèce dans le De natura rerum. Les ressemblances sont nombreuses: solennité, forme versifiée, apostrophe à Volupté-Vénus, écriture de l'éloge, thème de l'aimant universel et par conséquent du désir comme moteur de la vie et, derrière cela surtout, conception anti-métaphysique du monde : le bonheur apparaît comme à portée de l'homme. Mais la volupté y apparait aussi comme muse inspiratrice du poète (comme chez Lucrèce qui ouvre son long poème par cet hymne) : le plaisir est même conçu comme critère esthétique. Son conte s'achève ainsi sur l'éloge du «plus bel esprit de Grèce» et sur une prise de position à la fois épicurienne et poétique. On voit là combien la lecture intertextuelle nourrit l'interprétation du conte.

Un travail d'écriture peut permettre de rendre plus tangibles ces dialogues intellectuels entre époques et les effets d'entrelacement de plusieurs hypotextes. Il s'agira, par exemple, de réécrire une fable d'Ésope en lui donnant une dimension épicurienne (avec l'apport de textes épicuriens tels que la fameuse lettre d'Épicure à Ménécée sur la mort, ou bien l’hymne à Vénus de Lucrèce dans le De natura rerum). 


\section{Réécriture et histoire des genres}

L'histoire des genres relève également de mécanismes intertextuels au sens large. On peut considérer, par exemple, que la naissance de la tragédie grecque est fondamentalement intertextuelle: la plupart des tragédies grecques du $v^{e}$ siècle avant J.-C. sont en effet des amplifications de passages d'épopées faisant allusion à tel ou tel mythe. Edipe Roi a pour hypotexte quelques vers homériques et se présente comme la transposition d'un récit en dialogue théâtral, autrement dit, la transformation d'une «mimésis directe», selon les termes d'Aristote, en une "mimésis indirecte». Il en va de même des mystères médiévaux, réécriture de passages choisis de la Bible ou bien du genre des miracles, qui n'est autre que l'adaptation pour la scène de légendes de saints. Le travail en atelier d'écriture permet d'explorer ces phénomènes de transmodalisation et d'observer la manière dont les changements génériques influent sur l'écriture elle-même et orientent en profondeur le sens.

Il parait également fructueux d'aborder l'histoire du roman comme dialogue intertextuel, si l'on considère, à la suite de Bakhtine, que le roman «parodise» les autres genres. La reprise parodique des procédés propres à un genre aboutit au renouvellement des formes et à l'invention d'un nouveau genre, parfois reconnu comme tel longtemps après. Dans Les Testaments trahis ${ }^{14}$, Milan Kundera souligne le fait que les récits de géants de Rabelais n'étaient pas perçus comme des romans lors de leur publication. Ils le sont devenus du fait que de nombreux romanciers s'en sont ensuite explicitement réclamé : c'est ce phénomène d'intertextualité qui a fait de Rabelais, rétrospectivement, l'un des inventeurs du roman européen.

Comment exploiter cela en atelier d'écriture? Prenons l'exemple du récit des origines du géant Pantagruel (chapitre I), descendant d'une famille qui remonterait à l'absorption de «mesles», fruits monstrueux produits peu après le meurtre d'Abel par Caïn. Les références intertextuelles sont multiples : parodie de la Genèse, avec la référence à Caïn et Abel, puis au déluge et à l'arche de Noé; allusion aussi au début de l'Évangile de Mathieu, avec la parodie de la généalogie du Christ. Cette généalogie fantaisiste et le lien avec ses hypotextes bibliques permettent donc de comprendre non seulement comment fonctionne le comique chez Rabelais, mais aussi comment se crée le nouveau genre qu'invente Rabelais (qui lui-même s'inspire de Lucien) et ce qu'il doit à la parodie. Mais surtout, le fait de se frotter soi-même à l'écriture parodique peut permettre de saisir les enjeux de cette parodie: source de

I4. Gallimard, I993. 
comique, de désacralisation de textes sacrés, dans la tradition carnavalesque du monde à l'envers, où tout est permis, même de se moquer des Écritures saintes, mais aussi, et conjointement sans doute, hommage à ces textes et réinterprétation de leur sens. On peut alors saisir, par le biais de l'intertextualité, les liens qui unissent à travers l'histoire un ensemble de romans parodiques, à forte dimension critique, de Cyrano de Bergerac à Céline, en passant par Diderot ou Flaubert.

\section{Pastiche et histoire des mouvements littéraires}

Le pastiche n'est pas seulement, comme le disait Proust, une manière de faire de la critique littéraire en acte. Il peut être aussi une façon d'enseigner l'histoire des mouvements littéraires. Si l'on définit les pastiches, à la suite de Gérard Genette, comme imitation indirecte, non d'un texte précis, mais d'un ensemble de textes dont on dégage les caractéristiques, stylistiques autant que thématiques, on constate qu'ils ne se réduisent pas aux seuls pastiches d'écrivains. Les pastiches de mouvements littéraires, plus nombreux qu'on ne le pense à l'intérieur des œuvres littéraires ${ }^{15}$, ont le grand intérêt de présenter une sorte de comble des traits stylistiques et thématiques propres à ces mouvements.

Prenons l'exemple du propre pastiche que Du Bellay a fait du pétrarquisme dans son poème "Contre les pétrarquistes», poème d'autant plus intéressant qu'il représente une sorte d'auto-pastiche : le poète de la Pléiade se moque également de lui-même et de sa "période pétrarquiste» en énumérant toutes les images hyperboliques et stéréotypées reprises à Pétrarque pour évoquer la beauté divine de l'être aimé et les tortures infinies qu'endure l'amant parfait qu'est le poète. Faire en atelier d'écriture un pastiche d'écriture pétrarquiste, précieuse, romantique ou surréaliste exige au préalable d'avoir lu un ensemble de textes, repéré les manières d'écrire récurrentes, les motifs, les topoï et les genres de prédilection propres à ces mouvements. Un tel pastiche suppose en un mot d'être d'abord un lecteur attentif à la spécificité d'une écriture et à son inscription dans un contexte historique et culturel particulier. À terme, le fait d'avoir écrit à la manière des romantiques permet ensuite de repérer dans un texte littéraire non romantique, comme chez Flaubert ou Villiers de l'Isle Adam, des pastiches romantiques disséminés et de saisir leur signification ironique.

I 5. Songeons au pastiche de Montaigne chez La Bruyère, aux pastiches précieux chez Molière ou La Fontaine, aux pastiches romantiques chez Flaubert, au pastiche du style artiste des Goncourt chez Proust... 
Dans tous les cas, il s'agit bien d'appréhender par l'expérience d'écriture (et de lecture conjointe) la part de filiation littéraire et d'historicité de toute production littéraire.

\section{Réflexivité et réécriture de soi : de l'écriture littéraire à l'écriture critique}

Mais pour atteindre l'objectif ambitieux de faire de l'histoire et de la théorie littéraires en acte, il est indispensable d'introduire, à l'intérieur de l'atelier d'écriture, une dimension de réflexivité. Autrement dit, l'atelier d'écriture doit mettre chaque étudiant en posture de lecteur vigilant et actif, aussi bien par rapport à lui-même que par rapport aux autres participants. L'enseignant a dans cette optique un rôle essentiel à jouer puisque c'est d'abord à lui de réagir aux lectures, de donner des conseils pour aller plus loin, améliorer ou accentuer tel ou tel aspect et indiquer de quelle manière on peut aller au-delà de la première version écrite en séance.

C'est dans cette perspective qu'une séance est consacrée en début d'atelier aux brouillons d'écrivains : elle met l'accent sur l'importance de la réécriture et la manière de retravailler ses écrits. On part de l'analyse de manuscrits d'écrivains apportés par les étudiants et la séance est consacrée à travailler sur les quatre opérations principales de la récriture de soi (substitution, déplacement, amplification ou réduction).

Assez vite, une fois instaurée une règle de fonctionnement essentielle, l'écoute bienveillante du groupe, une fois les premières angoisses surmontées à l'idée de livrer ses textes en public, on peut amener les étudiants à réagir à leur tour aux écrits des autres. Il peut être bénéfique parfois de désigner pour chaque texte lu un lecteur privilégié différent qui sait par avance qu'il devra réagir, s'interroger sur la conformité du texte par rapport à la consigne proposée (sachant qu'il est toujours possible de transgresser la consigne, à condition d'en être conscient) et donner des conseils de réécriture.

Cette même posture critique est demandée à chaque étudiant à l'égard de ses propres écrits, au moment où il recopie «au propre», le plus souvent grâce à un traitement de texte, tous les textes qu'il a produits, en cours et en dehors des cours.

Ce sont ces commentaires critiques qui permettent de découvrir des effets inattendus du dispositif d'écriture mis en place.

Beaucoup d'étudiants insistent, par exemple, sur le rôle du groupe dans l'orientation des secondes versions : celles-ci, disent-ils, sont souvent inspirées par les textes des autres. Ils osent alors aller plus loin, se livrer davantage ou au 
contraire accentuer la dimension comique, transgresser davantage la consigne ou, au contraire, la suivre de plus près. Parfois ce sont les réactions des autres étudiants à leur lecture (rires, contresens éventuels, commentaires divers...) qui les amènent à réorienter, préciser ou accentuer la deuxième version. Autrement dit, le dialogue intertextuel joue également entre eux, à l'intérieur de l'atelier et le concept de "productivité» prend alors tout son sens. Beaucoup disent combien le groupe est porteur, suscite le désir d'écrire, au point qu'ils ont conscience parfois d'écrire pour les autres : «Durant ce cours, nous nous nourrissons les uns des autres», écrit une étudiante. «L'interprétation de chacun enrichit notre lecture. Nos créations sont le fruit d'un travail en commun.»

Parmi les bénéfices seconds, on note également une étonnante assurance à l'oral prise par les étudiants, dont certains avouent qu'ils hésitaient à participer à l'atelier essentiellement par hantise d'avoir à lire leurs textes devant d'autres. Progressivement, par une sorte d'émulation du groupe, cette épreuve se transforme en plaisir. Au lieu d'être lus à toute allure, les textes sont dits avec plus de force et de conviction, partagés, voire théâtralisés, et la séance consacrée à l'écriture théâtrale à deux devient alors possible.

Tous insistent sur la qualité humaine des relations qui s'instaurent dans le groupe, y compris entre des étudiants d'âges très divers. L'écriture ainsi partagée permet en quelques séances une connaissance des autres beaucoup plus intense et profonde que deux années universitaires. Mais l'écriture amène aussi les étudiants à découvrir leur propre écriture, à se découvrir ou s'étonner eux-mêmes, à prendre conscience de certains stéréotypes, à oser se lancer dans des registres ou genres nouveaux.

Enfin, le travail de réflexion critique rend possible le retour à une écriture critique, remotivée en quelque sorte et de meilleure qualité. Certaines propositions d'écriture amènent à s'apercevoir de la frontière parfois ténue entre fiction et commentaire : lors de la séance consacrée à la traduction, les étudiants prennent conscience, en transposant une page de Montaigne en français contemporain, que la traduction amène à interpréter, que la paraphrase elle aussi engage l'interprétation et que l'amplification comporte une part d'invention. Et lorsqu'ils font une deuxième version de Montaigne "pour la jeunesse», l'essai emprunte souvent la forme du conte ou de la parabole et l'on bascule à nouveau dans la fiction. Mais surtout, le fait de s'autoriser à porter un jugement sur les écrits de ses compagnons d'écriture est un tremplin pour pouvoir ensuite s'autoriser à porter un jugement sur des œuvres littéraires et les interpréter, ce qui, on le sait bien, même à l'université, n'est pas si fréquent que cela. La tendance est souvent de se réfugier derrière les ouvrages critiques qui précisément font autorité. 
«Entre bouillonnement, fièvre et doute, nous avons pénétré le processus de création", écrivait une étudiante dans son bilan de fin de semestre. L'expérience d'écriture en atelier est en effet précieuse, j'oserais même dire indispensable, pour pénétrer le processus de création. Il ne s'agit pas bien sûr de donner l'illusion à tous les étudiants qu'une carrière d'écrivain s'ouvre à eux, mais de leur permettre d'écrire mieux, d'utiliser l'écriture pour euxmêmes, pour mieux penser, pour se connaittre, pour mieux comprendre et apprécier la littérature.

L'écriture sous contrainte, d'appropriation et de détournement des textes vous conduit où vous ne pensiez pas aller, vous invite à découvrir des genres, des registres qui vous semblaient étrangers ou impossibles. Elle amène à lutter contre les stéréotypes et à parler de soi d'une manière paradoxalement beaucoup plus libre. Elle permet donc de percevoir la dimension fondamentalement oblique de la littérature : on parle de soi en empruntant la langue et les mots des autres, que l'on tente de s'approprier. Elle permet enfin de libérer la lecture en même temps qu'elle libère l'écriture. L'observation des étudiants m'amène à partager pleinement cette intuition de Roland Barthes :

Pour moi, ma conviction profonde et constante est qu'il ne sera jamais possible de libérer la lecture si, d'un même mouvement, nous ne libérons pas l'écriture ${ }^{16}$.

\section{Bibliographie complémentaire}

Armand A., L'histoire littéraire, théories et pratiques, Toulouse, Bertrand-Lacoste, CRDP

Midi-Pyrénées, I 993.

Bakhtine M., La Poétique de Dostö̈evski, Seuil, i 970, trad. de I. Kolitcheff.

-, L'cuvre de François Rabelais et la culture populaire au Moyen-Âge et sous la Renaissance,

Moscou, I965, Gallimard, I970, trad. de A. Robel.

-, Esthétique et théorie du roman, Moscou, I975, trad. de D. Olivier, Gallimard, I 978.

-, Esthétique de la création verbale, Moscou, I979, trad. de A. Aucouturier, Gallimard, I 984 .

Bauchau H., L'Écriture à l'éconte, Actes Sud, 2000.

Biasi P.-M. de, «Intertextualité (théorie de)», Encyclopaedia universalis, 1975.

Boissinot A., Littérature et histoire, Toulouse, Bertrand-Lacoste, I 998.

Bon F., Tous les mots sont adultes. Méthode pour l'atelier d'écriture, Fayard, 2000.

Compagnon A., La seconde main ou le travail de la citation, Seuil, I 979.

Costa P., Petit manuel pour écrire des haïku, Philippe Picquier, 2000.

Genette G., Palimpsestes, Seuil, I 982.

I6. R. Barthes, «Sur la lecture», Le Bruissement de la langue, Seuil «Points», I984, p. 46. 
Hamon P., L'Ironie littéraire. Essai sur les formes de l'écriture oblique, Hachette Supérieur, 1996.

Houdart-Mérot V., «Mise en perspective historique», Perspectives actuelles de l'enseignement du français, MEN, Direction de l'enseignement scolaire, CRDP de l'académie de Versailles, 200 I.

- «Un mythe et sa réécriture : la descente aux enfers» Des textes à l'cuure, Français I ${ }^{r e}$, Lancrey-Javal R. dir., Hachette-Éducation, 2001, p. 362-383.

- «Bakhtine, père ou ancêtre de l'intertextualité?», Regards sur l'héritage de Mikhaïl Bakhtine, CRTH, université de Cergy-Pontoise, contributions au séminaire annuel du CRTH 2002-2003, réunies par P.-P. Haillet, 2005.

- «Proust Dormeur éveillé, ou comment surseoir à l'arrêt de mort», Les Mille et Une nuits dans l'imaginaire $d u X X^{e}$ siècle, Chaulet-Achour Chr. coord., L'Harmattan (Études transnationales francophones et comparées), 2005.

- «Le pastiche de genre, entre feinte et réflexivité», Poétiques de la parodie et du pastiche de I850 à nos jours, Dousteyssier-Khoze C. et Place-Verghnes Fl. éd., Actes du colloque international de l'université de Durham, Éditions Peter Lang, 2006.

Oulipo, La Littérature potentielle, Gallimard «Idées», I 973.

Piegay-Gros N., Introduction à l'intertextualité, Dunod, I 996.

Pimet O., Boniface CL., Ateliers d'écriture, mode d'emploi, ESF, I 999.

Riffaterre M., "La syllepse intertextuelle», Poétique, n 40, nov. I979.

Sperger D., Wilson D., «Les ironies comme mentions», Poétique, n 36, Seuil, I 978.

Todorov T., Mikhaïl Bakhtine, le principe dialogique, Seuil, i 98 I. 